\title{
On the role of dislocation loops in silicon light emitting diodes
}

\author{
M. A. Lourenço, ${ }^{a)}$ M. Milosavljević, R. M. Gwilliam, and K. P. Homewood \\ Advanced Technology Institute, School of Electronics and Physical Sciences, University of Surrey, \\ Guildford, Surrey GU2 7XH, United Kingdom
}

\author{
G. Shao \\ BCAST, Brunel University, Uxbridge UB8 3PH, United Kingdom
}

(Received 7 July 2005; accepted 20 September 2005; published online 8 November 2005)

\begin{abstract}
The role of boron-induced dislocation loops on the suppression of the luminescence thermal quenching in silicon-based light-emitting diodes is investigated here. Luminescence measurements and cross-sectional transmission-electron-microscopy images from devices fabricated by boron implantation into crystalline silicon, and into a pre-amorphized substrate, to prevent the boron-induced loops formation, were compared. The results show that, in the devices incorporating dislocation loops between the depletion region and sample surface (the boron induced loops), the thermal quenching has been completely eliminated, in contrast with devices fabricated from the pre-amorphized substrate where strong thermal quenching is still observed. (C) 2005 American Institute of Physics. [DOI: 10.1063/1.2130533]
\end{abstract}

Silicon is an indirect band gap semiconductor and is fundamentally unable to emit light efficiently. Nevertheless, silicon is the basis of the microelectronic industry and several approaches have been considered to enhance its poor light emission. These include porous silicon, ${ }^{1}$ silicon-silicon dioxide superlattices, ${ }^{2}$ silicon nanoprecipitates in silicon dioxide, ${ }^{3}$ erbium in silicon, ${ }^{4}$ silicon/germanium, ${ }^{5}$ and iron disilicide. ${ }^{6}$ A common problem found in all these approaches is the strong thermal quenching leading to very poor performance at room temperature. $\mathrm{Ng}$ et al. $^{7}$ proposed a unique solution, the dislocation engineering method, to suppress the thermal quenching in semiconductor systems. The basis of the dislocation engineered approach is the controlled introduction, in a $p$ - $n$ junction between the depletion region and the surface, of dislocation loops. The loops modify the silicon band gap, on the $p$-side, to provide spatial confinement of charge carriers. Thus, diffusion of injected carriers to point defects and the surface, where efficient nonradiative recombination occurs, is minimized or eliminated. This approach was used, for example, to fabricate $\beta$-FeSi ${ }_{2}$ light emitting devices $(\mathrm{LEDs})^{8}$ and reduced the luminescence thermal quenching to only one order of magnitude of that of the low temperature emission, making these devices much more efficient then conventional ones, where room temperature luminescence was practically absent.

In this paper we present a detailed study of the role of the dislocation loops on the suppression of the luminescence thermal quenching. In order to directly compare the luminescence of devices with and without the incorporation of dislocation loops above the $p$ - $n$ junction, two batches of samples were designed. Devices were fabricated by boron implantation $\left(10^{15} \mathrm{~B} \mathrm{~cm}^{-2}\right.$ at $\left.30 \mathrm{keV}\right)$ into either a standard [100] $n$-type silicon substrate, phosphorous doped $(2-7 \Omega \mathrm{cm})$ or on an otherwise identical substrate but previously amorphized by a high energy silicon implantation $\left(6 \times 10^{15} \mathrm{Si} \mathrm{cm}^{-2}\right.$ at $\left.400 \mathrm{keV}\right)$. Thus, boron induced dislocation loops were formed above the junction in the crystalline substrate, but not in the pre-amorphized material. In both

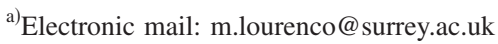

cases, the boron implantation provided the $p$-type dopant to form the $p-n$ junction. Subsequent to the boron implantation, all samples were annealed at $950{ }^{\circ} \mathrm{C}$ for $20 \mathrm{~min}$ in $\mathrm{N}_{2}$ ambient. Fabrication of light emitting devices, in both cases, was completed by deposition of front and back contacts and mesa etching, as described previously. ${ }^{8}$ Devices fabricated from the substrate containing the dislocation loops will be referred to as dislocation engineered light emitting diodes (DELEDs) and the ones fabricated from the pre-amorphized material will be referred to as conventional devices. The devices were characterized by electroluminescence (EL), photoluminescence (PL), and cross-sectional transmission electron microscopy (X-TEM). The EL and PL experiments were performed in the temperature range $80-300 \mathrm{~K}$, as described in Ref. 9. PL measurements were done at $100 \mathrm{~mW}$ using the $488 \mathrm{~nm}$ line of an Ar laser. EL measurements were performed under forward bias (current density of $3 \mathrm{~A} / \mathrm{cm}^{-2}$ ) extracting the light through a window at the back of the samples. Current-voltage characteristics were measured prior to the EL experiments and, in both cases, showed good diode characteristics, with a turn-on voltage of less than $1 \mathrm{~V}$. The basic procedure for the XTEM measurements is described in Ref. 10.

Cross-sectional TEM analysis after boron implantation and annealing is presented in Fig. 1. It shows that boron induced dislocation loops are present only in samples fabricated from the crystalline substrate; implantation of boron in pre-amorphized substrate does not induce the formation of dislocation loops. Figure 1(a) shows the X-TEM image of samples fabricated from the crystalline substrate. It can be seen that dislocation loops are located at a depth of $\sim 140 \mathrm{~nm}$, above the depletion region edge at $\sim 350 \mathrm{~nm}$. This is in agreement with the predicted ion range (1091 $\AA$ ) and straggle (394 ̊) for $30 \mathrm{keV} \mathrm{B} \mathrm{implantation} \mathrm{into} \mathrm{Si}^{11}$ These loops are extrinsic, either faulted or perfect, lying in \{111\} Si habit planes as described earlier. ${ }^{10}$ Figure 1(b) shows X-TEM image taken from samples fabricated from the pre-amorphized substrate. In this case only end of range (EOR) dislocation loops with respect to $\mathrm{Si}$ ion range are present. These are smaller loops and located much deeper in 

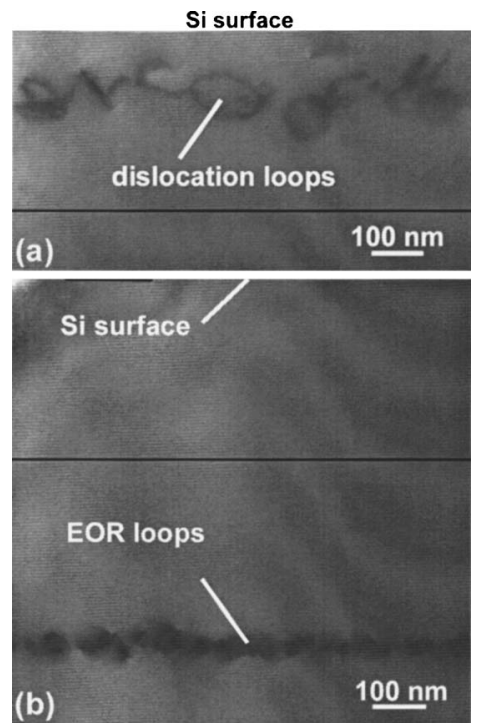

FIG. 1. Bright field X-TEM images taken near [110] Si of (a) the sample with dislocation loops induced directly by boron implantation, and (b) the pre-amorphized sample by implantation of silicon. The horizontal lines added to the figures illustrate the position of the depletion region edge on the upper, $p$-side, of the junction.

the substrate. They are also extrinsic in nature, faulted or perfect, situated at a depth of $\sim 700 \mathrm{~nm}$ from the Si surface, in accordance to the theoretical range $(6092 \AA)$ and straggle (1458 ̊) for $400 \mathrm{keV}$ Si ions implanted into $\mathrm{Si}^{11}$

Figure 2 shows the room temperature photoluminescence spectra of samples with and without dislocation loops. Room temperature PL is observed only from the sample containing dislocation loops. Figure 3 shows the EL measured at $80 \mathrm{~K}$ and at $300 \mathrm{~K}$ for both types of devices. The EL response for the dislocation engineered LED is dominated, at low temperatures, by a strong, narrow line peaked at $1.1 \mu \mathrm{m}$, corresponding to the $\mathrm{Si}$ band-edge emission. Emission at $1.1 \mu \mathrm{m}$ is practically absent in the conventional device at $80 \mathrm{~K}$, its EL response being dominated by a broad line in the region 1.3-1.7 $\mu \mathrm{m}$. At room temperature, EL at the silicon band edge is observed from both devices. However, the DELEDs are ten times brighter than the conventional devices. Figure 4 shows the EL integrated intensity plotted against measurement temperature for a DELED and a conventional device. The EL integrated intensity increases with

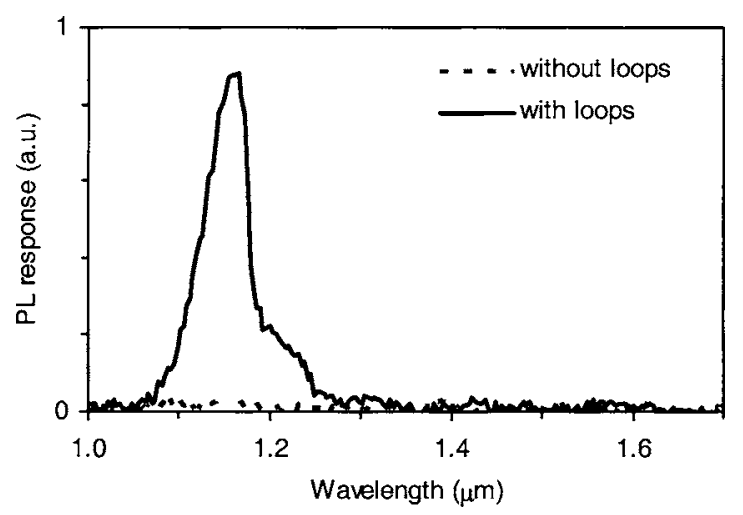

FIG. 2. Room temperature photoluminescence from samples with boron induced dislocation loops (continuous line) and without the boron induced dislocation loops (dashed line). dislocation loops (dashed line).
Downloaded 30 Mar 2009 to 131.227 .178 .132 . Redistribution subject to AIP license or copyright; see http:
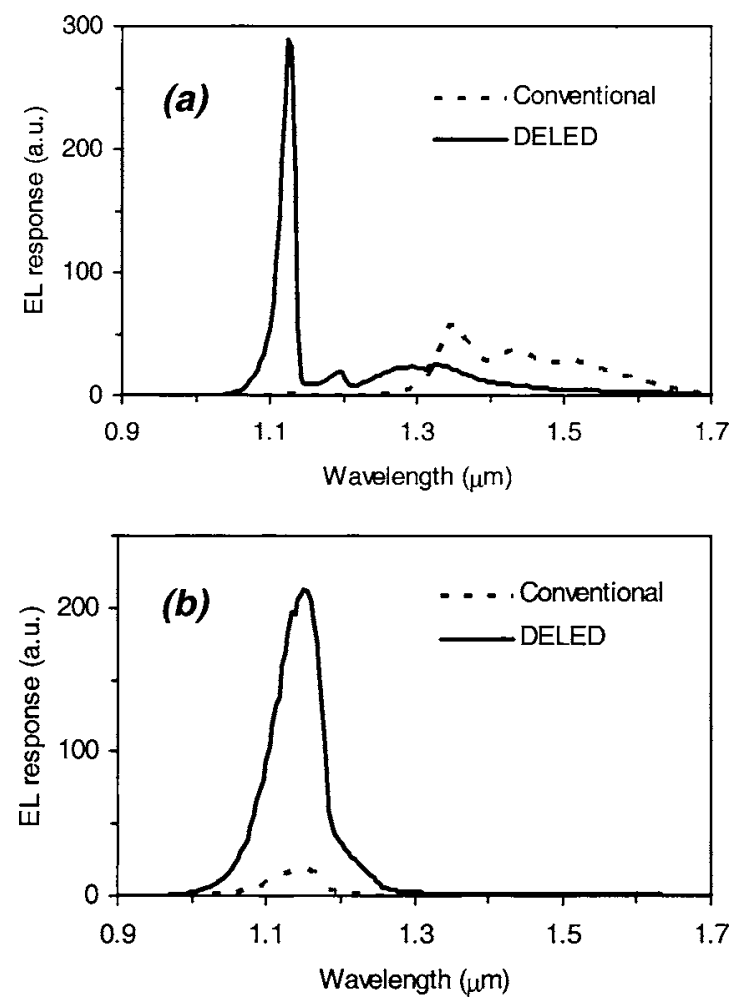

FIG. 3. Electroluminescence of a conventional and a dislocation engineered light emitting diode measured at (a) $80 \mathrm{~K}$ and (b) $300 \mathrm{~K}$. Devices were operated under forward bias at a constant current density of $3 \mathrm{~A} / \mathrm{cm}^{2}$.

temperature for the DELED, and decreases for the conventional device.

The increase of EL integrated intensity with temperature observed only in the dislocation engineered light emitting diodes clearly shows that the incorporation of boron induced dislocation loops between the device active region and surface is responsible for the elimination of the luminescence thermal quenching. The dislocation loop array introduces a strain field outside the loop that increases the silicon band gap by up to $0.75 \mathrm{eV}^{7}$ and enables spatial confinement of the injected carries, so carrier diffusion to point defects and the surface, where efficient non-radiative recombination occurs, is suppressed. Dislocation loops above the junction were not formed by boron implantation into the pre-amorphized substrate. Indeed, only Si induced end-of-range dislocations, located much deeper in the substrate, could be observed, so

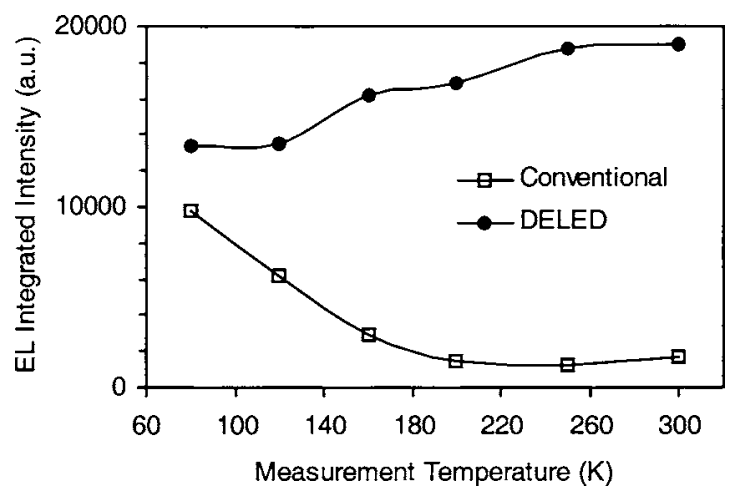

FIG. 4. Room temperature electroluminescence integrated intensity as a function of measurement temperature for a conventional and a dislocation engineered light emitting diode. Devices were operated under forward bias 
spatial confinement of charge carriers to prevent nonradiative recombination was absent. These end of range dislocations are probably the origin of the EL emissions observed at $80 \mathrm{~K}$. These lines, at $0.82,0.86$, and $0.92 \mathrm{eV}$, are the D1, D2, and D3 lines, commonly reported to appear in ion implanted silicon. $^{12}$ They are also thought to be related to the small increment in the silicon integrated intensity with temperature observed in the pre-amorphized devices. In this situation, the silicon emission is fed from the emission from the $D$ lines. Indeed, an increase in silicon band edge emission due to a simultaneous decrease in other centers has been observed and successfully modelled before. ${ }^{8}$

The external quantum efficiencies collected, just through the windows, of the devices into a hemisphere are $0.02 \%$ for the dislocation engineered LED and $0.002 \%$ for the conventional silicon LED. It should be noted that, because these are planar devices, the measured total external quantum efficiency is limited by total internal reflection that reduces the extraction efficiency through the front surface by a factor of $\sim 60$ of the total EL produced internally. This effect is simply the result of geometric optics and could be largely eliminated by commercial LED packaging or by surface texturing, as in the case of the silicon diode reported by Green et al. ${ }^{13}{ }^{3}$ where an external quantum efficiency of $\sim 1 \%$ in high purity silicon devices was reported. In general, the quantum efficiency in a LED is a function of injection current ${ }^{14}$ because competing nonradiative routes, in particular Schockley-Hall-Reed (SHR) recombination, through bulk and surface defects at low injection currents, and Auger recombination at very high injection. The EL of the dislocation engineered devices reported here is found to be linear up to the maximum current densities measured of $15 \mathrm{~A} / \mathrm{cm}^{2}$. This is consistent for a low to medium injection regime where, as postulated here, recombination through defects (the SHR route) have been decoupled from the radiative route and before the onset of significant Auger recombination.

In summary, we have demonstrated here that the controlled introduction of dislocation loops, to allow their location, mean size and density to be engineered, is responsible for the suppression of the luminescence thermal quenching previously observed in silicon-based light emitting diodes.
Dislocation engineered light emitting diodes are efficient at room temperature and generally brighter than at lower temperatures. In contrast, thermal quenching is strong in devices fabricated without the introduction of dislocation loops into the active region of the device. It is the stress field introduced by the array of dislocation loops that creates a blocking potential that, when placed adjacent to and just above the depletion region edge, prevents carrier diffusion to point defects and surface, where efficient nonradiative recombination occurs, so radiative recombination is enhanced. Thus, by decoupling the nonradiative recombination routes, the luminescence thermal quenching can be suppressed or eliminated, as band-to-band transitions are relatively temperature independent, in contrast to nonradiative routes which show strong temperature dependence.

${ }^{1}$ K. D. Hirschman, L. Tysbekov, S. P. Duttagupta, and P. M. Fauchet, Nature (London) 384, 338 (1996).

${ }^{2}$ Z. H. Lu, D. J. Lockwood, and J. M. Baribeau, Nature (London) 378, 258 (1995).

${ }^{3}$ T. Komoda, J. Kelly, F. Cristiano, A. Nejim, P. L. F. Hemment, K. P. Homewood, R. Gwilliam, J. E. Mynard, and B. J. Sealy, Nucl. Instrum. Methods Phys. Res. B 96, 387 (1995).

${ }^{4}$ B. Zheng, J. Michel, F. Y. G. Ren, L. C. Kimerling, D. C. Jacobson, and J. M. Poate, Appl. Phys. Lett. 64, 2842 (1994).

${ }^{5}$ L. Vescan and T. Stoica, J. Lumin. 80, 485 (1999).

${ }^{6}$ D. Leong, M. Harry, K. J. Reeson, and K. P. Homewood, Nature (London) 387, 686 (1997).

${ }^{7}$ W. L. Ng, M. A. Lourenço, R. M. Gwilliam, S. Ledain, G. Shao, and K. P. Homewood, Nature (London) 410, 192 (2001).

${ }^{8}$ M. A. Lourenço, M. Milosavljevic, R. M. Gwilliam, G. Shao, and K. P. Homewood, Thin Solid Films 461, 219 (2004).

${ }^{9}$ M. A. Lourenço, M. Milosavljevic, S. Galata, M. S. A. Siddiqui, G. Shao, R. M. Gwilliam, and K. P. Homewood, Vacuum 78, 551 (2005).

${ }^{10}$ M. Milosavljevic, G. Shao, M. A. Lourenço, R. M. Gwilliam, and K. P. Homewood, J. Appl. Phys. 97, 073512 (2005).

${ }^{11}$ J. F. Ziegler, J. P. Biersak, and U. Littmark, The Stopping and Range of Ions in Solids (Pergamon, New York, 1985).

${ }^{12}$ R. Sauer, J. Weber, J. Stolz, E. R. Weber, K.-H. Küsters, and H. Alexander, Appl. Phys. A: Solids Surf. 36, 1 (1985).

${ }^{13}$ M. A. Green, J. Zhao, A. Wang, P. J. Reece, and M. Gal, Nature (London) 412, 805 (2001).

${ }^{14}$ T. Stoica, L. Vescan, A. Mück, B. Holländer, and G. Schöpe, Physica E (Amsterdam) 16, 359 (2003). 